\title{
Forcing and Type Theory
}

\author{
Thierry Coquand \\ Department of Computer Science and Engineering, \\ Göteborg University and Chalmers University of Technology, Sweden \\ coquand@chalmers.se
}

We present the technique of forcing from a constructive point of view. The connections with proof theory [1] and constructive mathematics are not so surprising given that Cohen's original discovery of forcing was "motivated by an attempt to prove analysis consistent" and the idea that statements which seemed to involve infinities "could be reduced to pieces of finite informations" [9]. The interest of combining forcing and realizability was pointed out in the proof of Goodman's Theorem [5].

We explain first how forcing allows to explain non effective objects (such as non principal ultrafilters, or well-ordering of the reals) in term of classical logic and dependent choices [7. The combination of classical logic and dependent choices have a computational interpretation 346]. However, the associated computational behaviour is difficult to describe. We show then how to describe in type theory the addition of one Cohen real. We make explicit the computations involved in such an extension. One application, similar to the ones described in the reference [2], is the uniform continuity of functionals on Cantor space definable in type theory. By iterating this extension, we get a computational interpretation of universal quantification over Cantor space.

\section{References}

1. Avigad, J.: Forcing in proof theory. Bulletin of Symbolic Logic 10(3), 305-333 (2004)

2. Beeson, M.: Foundations of constructive mathematics. Metamathematical studies. In: Ergebnisse der Mathematik und ihrer Grenzgebiete (3) [Results in Mathematics and Related Areas (3)], vol. 6, Springer, Berlin (1985)

3. Berardi, S., Bezem, M., Coquand, T.: On the Computational Content of the Axiom of Choice. J. Symb. Log. 63(2), 600-622 (1998)

4. Berger, U., Oliva, P.: Modified bar recursion. Mathematical Structures in Computer Science 16(2), 163-183 (2006)

5. Goodman, N.: Relativized realizability in intuitionistic arithmetic of all finite types. J. Symbolic Logic 43(1), 23-44 (1978)

6. Krivine, J.L.: Dependent choice, 'quote' and the clock. Th. Comp. Sc. 308, 259-276 (2003)

7. Krivine, J.L.: Structures de réalisabilité, RAM et ultrafiltre sur $\mathbb{N}$ (to appear, 2009)

8. Martin-Löf, P.: Constructive mathematics and computer programming. In: Logic, methodology and philosophy of science, VI (Hannover, 1979). Stud. Logic Found. Math., vol. 104, pp. 153-175. North-Holland, Amsterdam (1982)

9. Platek, R.: Kreisel, Generalized Recursion Theory, Stanford and Me. In: Odifreddi, P. (ed.) Kreiseliana, About and Around Georg Kreisel (1996) 